\title{
LOS DERECHOS DE LA LIBRE EXPRESIÓN EN LA POLÍTICA UNIFORME DE RESOLUCIÓN DE CONTROVERSIAS DE LA ICANN
}

\author{
A MISAPPLICATION OF FREE SPEECH RIGHTS IN THE ICANN'S \\ UNIFORM DISPUTE RESOLUTION POLICY
}

\begin{abstract}
JELENA LAKETiĆ*
RESUMEN: Este artículo tiene por objetivo analizar de qué manera la aplicación de la Política Uniforme, un mecanismo único para la resolución de controversias entre los nombres de dominio de internet y las marcas comerciales a nivel internacional, se ha convertido en un instrumento para restringir e ignorar los derechos de la libre expresión en internet.
\end{abstract}

Palabras clave: Libertad de expresión, política uniforme, ICANN.

ABSTRACT: The purpose of this study is to gain a better understanding of how the application of the UDRP, a mechanism for resolving disputes between domain name registrants and trademark holders, has become an instrument to restrict freedom of expression over Internet.

Keywords: Freedom of speech, UDRP, ICANN.

\section{INTRODUCCIÓN}

Los debates contemporáneos sobre internet están enmarcados en el contexto económico, político y social, en el que se destacan tres áreas de preferencia: la seguridad nacional, la libertad de expresión y la protección de los derechos de la propiedad industrial. Últimamente, dichas polémicas se centran en la importancia de la libre expresión en línea, así como los riesgos de censura y vigilancia por parte de los gobiernos, lo que deja en estado de indefensión a los ciudadanos ${ }^{1}$.

Si bien estas discusiones son importantes, en lugar de evaluar normativamente las diferentes políticas de internet promulgadas por los Estados, este trabajo aborda el tema del incremento súbito de las amenazas a la libertad de expresión desde una perspectiva diferente, estableciendo el vínculo entre las marcas comerciales y la libertad de expresión.

A prima facie parecería que no existe una relación directa entre la propiedad industrial y la libertad de expresión que pueda tener consecuencias profundas, ya que las marcas tradicionalmente han desempeñado un rol más comercial que social.

\footnotetext{
Magíster en Derecho con Mención en Contratación Comparada e Internacional, Universidad de Chile y Graduate Certificate in International Contracts and Business Law por American University Washington College of Law. Dirección postal: 3039 Ellis Street, Apt B Berkeley CA, 94703 United States. Dirección electrónica: j.laketic@gmail.com

1 Powers y Jablonski (2015).
} 
Como es de conocimiento común, un titular de la marca comercial dispone de ius excludendi omnes alios, teniendo los derechos exclusivos, aunque limitados, a utilizar su marca, así como el derecho a impedir a otras personas su utilización. El propósito de la propiedad industrial es la protección del producto, servicio, establecimiento comercial o industrial específicamente protegido por el registro marcario y para productos o servicios similares o relacionados. Pese a que la procedencia histórica del derecho marcario suponía la protección de los productores de desviaciones ilegítimas de su comercio por parte de los competidores, el desarrollo posterior ha conducido a alcanzar también la protección al consumidor y a la competencia leal en los mercados ${ }^{2}$. Por la doctrina contemporánea se reconoce que la finalidad de la propiedad industrial es evitar la confusión de los consumidores con respecto a la procedencia de determinados productos o servicios, al proporcionar indicadores confiables de las fuentes de productos y servicios en el mercado ${ }^{3}$. Asimismo, se ha señalado que el derecho marcario debe proteger a los productores de esos productos y servicios de la apropiación indebida de la buena voluntad de una marca, permitiendo así que las empresas se beneficien de una reputación de calidad bien ganada ${ }^{4}$. En general, la doctrina moderna destaca que estos objetivos deben funcionar en armonía 5 .

Por consiguiente, las marcas comerciales tienen una importancia significativa para la sociedad, ya que permiten resguardar el ámbito de producción de una firma y la competencia leal en el mercado. Asimismo, su función económica ayuda a reducir los costes de búsqueda de los consumidores y ayuda a mantener un mercado competitivo sin mayores distorsiones ${ }^{6}$.

Por otra parte, el derecho a la libre expresión es un derecho fundamental de todo individuo. La Declaración Universal de Derechos Humanos prevé que "todo individuo tiene derecho a la libertad de opinión y de expresión; este derecho incluye el no ser molestado a causa de sus opiniones, el de investigar y recibir informaciones y opiniones, y el de difundirlas, sin limitación de fronteras, por cualquier medio de expresión"7. Los tratados internacionales adoptados con posterioridad a la Declaración Universal también consagran el derecho a la libertad de pensamiento y de expresión. El Pacto Internacional de Derechos Civiles y Políticos en el artículo 19 establece que "nadie será molestado a causa de sus opiniones". Además, téngase en cuenta que aparte de su participación en el sistema universal de tratados de derechos humanos de las Naciones Unidas, los Estados también forman parte en los sistemas regionales de derechos humanos, lo que puede aumentar el nivel de la protección de las personas bajo su jurisdicción.

Nos parece pertinente destacar tres funciones del derecho a la libertad de expresión que nos permiten poner en evidencia su impacto en una sociedad democrática. En primer lugar, cabe señalar la dimensión individual y colectiva de este derecho. El aspecto individual protege a cada persona a pensar por sí misma y expresar autónomamente el propio

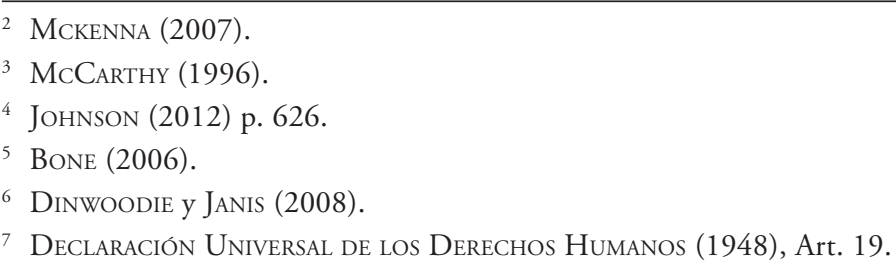


pensamiento, mientras que la dimensión colectiva implica el derecho "a recibir cualquier información y a conocer la expresión del pensamiento ajeno" en una agrupación ${ }^{8}$. En segundo lugar, en la jurisprudencia se subraya la dimensión instrumental de este derecho, ya que sirve como garantía del pleno goce y ejercicio de los demás derechos fundamentales? Finalmente, la libertad de expresión se impone como conditio sine qua non para garantizar el funcionamiento y la preservación del régimen democrático ${ }^{10}$.

Cabe destacar que antes de ser un atributo protegido por los ordenamientos jurídicos nacionales, la libertad de expresión igualmente es una categoría ética que ha de ser tenida en cuenta. Como tal, este derecho fundamental claramente está sujeto a las limitaciones y excepciones impulsadas por las concepciones religiosas, culturales, históricas, políticas, entre otras. Por esta razón, aunque se trata de un derecho universal, los contenidos y los límites de ese derecho varían en este orden de un Estado a otro.

Implícitamente, podemos entender que la plena aplicación de lo previsto dentro de la Declaración Universal de Derechos Humanos es posible únicamente en internet, ya que solo esta plataforma posibilita difundir las opiniones sin un intermediario, independientemente de las fronteras estatales y las posibles restricciones que estas suponen. Su desarrollo puede beneficiar el ejercicio de los derechos fundamentales, los procesos participativos y democráticos, así como las actividades sociales y comerciales. Del mismo modo, "a medida que internet se vuelve cada vez más esencial para las actividades cotidianas de las personas, la arquitectura tecnológica y las opciones de diseño incorporadas en aquella arquitectura tienen consecuencias cada vez mayores para los derechos humanos y el valor compartido" ${ }^{11}$.

Como se explicará a continuación, la gobernanza de internet carece de un organismo o régimen establecido por un tratado global. En lugar de ello, la gestión se realiza por organismos técnicos del sector privado que se dedican simplemente a mantener el sistema en funcionamiento. La gobernanza de estos órganos técnicos se concibe como un proceso de toma de decisiones impulsadas por parte de múltiples actores, como gobiernos, ingenieros, empresas, y sociedad civil. Teniendo esto en cuenta, hasta hace poco tiempo ningún organismo de carácter internacional se había pronunciado para establecer los requisitos que deben exigirse respecto a la libertad de expresión en internet. Un primer paso en esta dirección representa la resolución del Consejo de Derechos Humanos de Naciones Unidas, donde se afirma que "los derechos de las personas también deben estar protegidos en internet,

\footnotetext{
8 La colegiación obligatoria de periodistas (arts. 13 y 29 Convención Americana sobre Derechos Humanos), Opinión Consultiva OC-5/85 del 13 de noviembre de 1985. Serie A No 5, párrafo 31.

9 Claude Reyes y otros vs. Chile (Fondo, Reparaciones y Costas). Corte Interamericana de Derechos Humanos. Sentencia de 19 de septiembre de 2006, Serie C No 151, párr. 75.

10 Opinión Consultiva la Colegiación Obligatoria de Periodistas (arts. 13 y 29 Convención Americana sobre Derechos Humanos), supra nota 8, párr. 70; Corte IDH, CASO de Claude Reyes y otros, supra nota 9, párr. 85; Usón Ramírez vs. Venezuela, (Excepción Preliminar, Fondo, Reparaciones y Costas). C Corte Interamericana de derechos Humanos. Sentencia de 20 de noviembre de 2009, Serie C No 207, párr. 47; Ricardo Canese vs. Paraguay. Fondo, Reparaciones y Costas. Corte Interamericana de derechos Humanos. Sentencia de 31 de agosto de 2004. Serie C No 111, párr. 87.

11 "As the Internet is becoming ever more essential for individuals' everyday activities, the technological architecture and design choices embedded in them have ever greater consequences for human rights and shared values". ZALNIERIUTE y SCHNEIDER (2014) p. 5.
} 
en particular la libertad de expresión, que es aplicable sin consideración de fronteras y por cualquier procedimiento que se elija, de conformidad con el artículo 19 de la Declaración Universal de Derechos Humanos y del Pacto Internacional de Derechos Civiles y Políticos". Dicho documento reconoce la naturaleza global y abierta de internet y exhorta a los Estados a promover y facilitar el acceso a internet y la cooperación internacional ${ }^{12}$.

El Tribunal Europeo de Derechos Humanos ha reconocido que el conjunto de garantías generales consagradas a la libertad de expresión constituye una base adecuada para reconocer igualmente estas garantías en materia de libertad de expresión sin trabas a internet. En este orden de ideas, se ha destacado que "internet es en la actualidad el principal medio de la gente para ejercer su derecho a la libertad de expresión y de información: ofrece herramientas esenciales de participación en actividades y debates relativos a cuestiones políticas o de interés público"13.

Sin duda, la aparición de internet ha provocado una convergencia en la conformación de un nuevo contexto regulatorio: la solución de conflictos sobre los nombres de dominio de internet. Esto, dado que, en los últimos años, la propiedad industrial se encuentra perturbada por el desarrollo de las nuevas tecnologías y el uso de las marcas a través del internet. El uso de medios de comunicación ampliamente extendidos ha posibilitado las oportunidades para dañar a las marcas comerciales, facilitando la difusión de información engañosa de forma que pueda inducir a confusión respecto de la afiliación del titular de la marca, o a través de la comercialización externa de los productos y servicios protegidos por una marca, lo que se denomina como cybersquatting (ciberocupación).

Es importante entender que, en términos generales, un nombre de dominio representa la dirección de la red que posee una página web, personalizando la existencia en línea. Cabe decir que a través de la intervención del gobierno estadounidense se ha establecido la Corporación para la Asignación de Números y Nombres en Internet (ICANN), una organización sin fines de lucro ubicada en California para gobernar la coordinación de los vínculos entre las direcciones IP y nombres de dominio en todo internet. Esto significa que el principal propósito de la ICANN es coordinar el sistema de nombres de dominio (DNS), lo que permite traducir las direcciones IP (números que identifican un dispositivo en una red) de cada nodo activo en la red, a términos fáciles de memorizar y de manera que esos pueden comunicarse entre $s^{14}{ }^{14}$. La ICANN está basada en el singular modelo de múltiples partes interesadas denominado "multi-stakeholder", basado en la creencia de que las partes directamente involucradas en la gestión de los sistemas técnicos son las más capaces de ejecutar el sistema, tomando las decisiones basadas en la cooperación entre todos los actores interesados. Como lo ejemplifica la propia ICANN, dicho principio se basa en el principio de "consenso aproximado y código de ejecución". En lugar de voto formal, un consenso aproximado significa que una "gran mayoría de los que se preocupan debe estar de acuerdo",

\footnotetext{
12 Naciones Unidas, Asamblea General, Consejo de Derechos Humanos. Resolución sobre la promoción, protección y disfrute de los derechos humanos en Internet. UN Doc. A / HRC/20/L.13 (29 de junio de 2012).

13 Ahmet Yildirim c. Turquía, de 18 de diciembre de 2012, $\$ 31$ y 54.

${ }^{14}$ Mueller (2002).
} 
pero el método exacto para determinar el consenso puede variar de un grupo de trabajo a otro. La ejecución del código se basa en protocolos de prueba para ver si funcionan ${ }^{15}$.

Desde su creación, la ICANN se embarcó en una serie de movimientos diseñados a consolidar su autoridad, ampliando sus áreas de funcionamiento. En los últimos años, la ICANN ha aumentado en tamaño, principalmente debido a la introducción de nuevos dominios genéricos de nivel superior (gTLD) ${ }^{16}$.

Asimismo, su presencia ha inspirado una serie de publicaciones que desde una óptica crítica y multidisciplinar exponen diversos temas asociados con la existencia de esta entidad.

Mueller ha descrito en detalle el proceso de creación de la ICANN, así como trámites vinculados con su operación ${ }^{17}$. Numerosos académicos han apuntado que el desarrollo de la ICANN representa un ejemplo de innovación institucional, estableciendo un nuevo tipo de mecanismo regulatorio de carácter internacional ${ }^{18}$. También, se ha comentado de forma diversa sobre el establecimiento de la ICANN como un esfuerzo deficiente o un experimento audaz sin precedentes ${ }^{19}$. Varios académicos han examinado la cuestión de quién controla efectivamente a la ICANN ${ }^{20}$. Muchos han cuestionado la legitimidad del modelo de la ICANN en términos de democracia, consenso, la incapacidad de sacudir el dominio de Estados Unidos sobre internet y su base legal ${ }^{21}$. Se ha criticado la base contractual para gobernar la infraestructura crítica del internet ${ }^{22}$, así como la posición clave de la ICANN en términos de regulación internacional ${ }^{23}$.

Otras opiniones doctrinarias abordan los problemas causados en relación con la propiedad intelectual ${ }^{24}$, y temas de derechos humanos ${ }^{25}$. Finalmente, algunas fuentes doctrinales han examinado el modelo multi-stakeholder de la ICANN ${ }^{26}$.

Para dar sentido a las referencias anteriormente mencionadas sostenemos que, al ejercer el control sobre acceso a elementos críticos de la infraestructura técnica de internet, tales como el sistema que comprende los protocolos para enrutamiento, denominación y direccionamiento, junto con los estándares técnicos, la ICANN no se abstiene de la promulgación de políticas, cuyo ejemplo más notable es la promulgación de la Política Uniforme, la que desafía a la libertad de expresión. Este artículo profundiza la idea de que el derecho marcario se está distorsionando a través de dicha Política provocando serios

\footnotetext{
15 Hoffman (2012).

16 Un dominio de nivel superior genérico (gTLD) es una extensión de nombres de dominio con tres o más caracteres y no está asociado a países.

17 Mueller (2002).

18 Froomkin (2002); Mueller (2002).

19 KleinWÄchter (2000); WeinberG; (2000); Froomkin (2002).

20 Véase en particular Froomkin (2002); Weinberg (2000); Mueller (2002).

21 Mueller (2002); Froomkin (2002); Froomkin y Lemley (2003); Weinberg (2000); Simon (2012).

22 Bygrave (2015).

23 Mueller (2010).

24 Froomkin (2002); Dinwoodie y Janis (2008); Geist (2002); Lipton (2005, 2010); Woodard (2009).

25 Mueller (2010); Zalniurute y Schneider (2014), Lipton (2012), Nunziato (2003).

26 Hofmann (2016); Hofmann et al. (2017).
} 
inconvenientes para la libre expresión: ampliando los derechos de los titulares de la marca en base a la dilución, incorporando la polémica doctrina estadounidense de interés inicial de confusión, descartando la doctrina del uso leal, simplificando la prueba de confusión e ignorando el fenómeno de la ciberocupación inversa. Asimismo, las políticas de la ICANN sobre la aprobación de solicitudes para nuevos nombres de dominio de nivel superior genéricos amplían el alcance de la protección de la marca comercial más allá de lo previsto por las legislaciones nacionales.

\section{LIBERTAD DE EXPRESIÓN BAJO LA POLÍTICA UNIFORME}

Los creadores de la Política Uniforme de carácter global siempre han promovido este mecanismo como un enfoque equilibrado de resolución de controversias, basado en un mandato extremadamente limitado, ya que está diseñada exclusivamente para tratar los casos de ciberocupación. En cuanto a las controversias aplicables, la Política Uniforme se basa en tres circunstancias de carácter acumulativo:

a) cuando se trata de un nombre de dominio idéntico o similar hasta el punto de crear confusión con respecto a una marca de productos o servicios sobre la que el demandante tiene derechos;

b) cuando el registrante no tiene derechos o intereses legítimos respecto al nombre de dominio;

c) cuando el nombre de dominio ha sido registrado y está siendo utilizado de mala $\mathrm{fe}^{27}$.

La redacción de dicho mecanismo también aclara que la doble carga de la prueba recae sobre el demandante, ya que se requiere demostrar tanto el registro como la utilización de mala fe respecto al nombre de dominio en disputa.

En virtud del artículo 4 (c) se enumeran tres circunstancias que pueden servir para demostrar los derechos o intereses legítimos sobre el nombre de dominio del demandado:

1. antes de haber recibido cualquier aviso de la controversia, el demandado ha utilizado el nombre de dominio, o ha efectuado preparativos demostrables para su utilización, o un nombre correspondiente al nombre de dominio en relación con una oferta de buena fe de productos o servicios;

2. el demandado (en calidad de particular, empresa u otra organización) ha sido conocido corrientemente por el nombre de dominio, aun cuando no haya adquirido derechos de marcas de productos o de servicios;

3. el demandado hace un uso legitimo no comercial o un uso leal del nombre de dominio, sin intención de desviar a los consumidores de manera equivoca o de empañar el buen nombre de la marca de productos o de servicios en cuestión con ánimo de lucro.

\footnotetext{
${ }_{27}$ Véase el artículo 4 (a) de la Política Uniforme para la resolución de conflictos en materia de nombres de dominio.
} 
Esta última cláusula representa el único punto de relevancia para poder proteger la libertad de expresión, aunque en la práctica ha dado lugar a inconsistencias, como veremos en más detalle. Desde la entrada en vigor de la Política Uniforme, se ha argumentado que no se ha puesto atención adecuada a este requisito. En el año 2001, Froomkin ha argumentado que esta categoría representa a una extraña mezcla de conceptos incompatibles. Esto, porque se hace difícil determinar el uso comercial legítimo en estos casos, ya que la falta de intención de obtener beneficio comercial en este caso solo se puede referir a la difuminación de la marca ${ }^{28}$.

Sin embargo, no todas las jurisdicciones aplican la doctrina de la infracción a través de la dilución de la marca, que frecuentemente se aplica en el derecho estadounidense. Por consiguiente, incluso dentro de la propia ICANN se han expresado preocupaciones de que el concepto de la dilución por la degradación respecto al artículo 4 (c) (iii) de la Política Uniforme necesita más aclaración, ya que este instituto legal podría ser mal interpretado por aquellos que no están familiarizados con la legislación estadounidense y se podría aplicar de manera inapropiada para usos no comerciales de los nombres de dominio ${ }^{29}$.

En la legislación estadounidense, la dilución como la disminución de la capacidad de una marca famosa a identificar y distinguir bienes o servicios, independientemente de la presencia o ausencia de:

a) competencia entre el propietario de la marca famosa y otras partes,

b) riesgo de confusión, error o engaño.

Las acciones de dilución caen en una de las dos categorías. La primera es la dilución por difuminación (blurring), la cual se manifiesta como el debilitamiento del poder de venta y el valor de una marca a través del uso no autorizado de una marca de productos o servicios de naturaleza diferente, pero que puede debilitar las asociaciones entre una marca con su categoría de producto y otros aspectos distintivos, que entonces deja de funcionar como un identificador único ${ }^{30}$. La segunda es la dilución por degradación (tarnishment) y se produce cuando una marca famosa se vincula con productos o servicios dañinos o de mala calidad, creando asociaciones no deseadas, o causando una reducción del nivel de preferencia de la marca famosa ${ }^{31}$.

Como se puede observar, la dilución es una noción expansiva porque reconoce el daño al titular de una marca comercial independientemente de la confusión del consumidor, lo que no es compatible con los principios del derecho marcario que se basa en proteger a los consumidores de la confusión en cuanto a la fuente de productos o servicios particulares. En realidad, cualquier tipo de crítica puede diluir una marca.

\footnotetext{
28 Froomkin (2002) p. 662.

29 Véase Segundo Informe del Personal sobre los Documentos de Implementación de la Política de Resolución de Disputas de Nombres de Dominio Uniformes (24 de octubre de 1999).

3015 U.S.C. $\$ 1125$ (c) (2) (B).

$31 \quad 15$ U.S.C. $\$ 1125(\mathrm{c})(2)(\mathrm{C})$.
} 
No obstante, respecto al artículo 4 (c) (iii) de la Política Uniforme algunos Paneles han intentado establecer una explicación más detallada de circunstancias que servirán como prueba de los derechos o intereses legítimos del demandado sobre el nombre de dominio en disputa. Estas son las siguientes: (i) el nombre de dominio ha sido registrado y es usado únicamente con fines expresivos; (ii) el registrante del nombre de dominio cree que las críticas son fundadas y carece de intención de obtener ganancias comerciales; (iii) es evidente para los usuarios de internet que visitan el sitio web que el nombre de dominio no es operado por el titular de la marca; (iv) el demandado se ha abstenido de registrar todos o la mayoría de los nombres de dominio razonablemente adecuados para el titular de la marca; (v) se proporciona un enlace prominente y apropiado al sitio web del propietario de la marca comercial correspondiente; vi) cuando los correos electrónicos destinados al demandante utilicen el nombre de dominio en cuestión, se envía una alerta a los remitentes de manera adecuada para que sus correos electrónicos hayan sido mal dirigidos ${ }^{32}$.

Con respecto a esto, han surgido dos líneas en práctica. Una, donde el derecho de la libre expresión no se extiende necesariamente al registro y uso de un nombre de dominio que sea idéntico o confusamente similar a la marca registrada ${ }^{33}$. La segunda que protege el interés legítimo del demandado en usar la marca registrada como parte del nombre de dominio para fines expresivos, siempre cuando dicho uso es leal y no comercia ${ }^{34}$. Curiosamente, en los casos cuando se asignan panelistas de nacionalidad estadounidense a resolver la disputa, se tiende a adoptar este razonamiento ${ }^{35}$.

Estas tendencias opuestas amenazan el objetivo principal de la Política Uniforme, ya que son incompatibles con la uniformidad global que se pretende lograr.

\section{CYBERSQUATTING VERSUS CRÍTICA}

Dado que la Política Uniforme no siempre alcanza el equilibrio entre los intereses comerciales de la propiedad industrial y los derechos de la libre expresión, cabe aclarar que los nombres de dominio no se registran únicamente con fines especulativos.

En realidad, dentro de los sitios con fines expresivos es posible distinguir los sitios de discusión, los sitios de ajustes de cuentas (score-settling sites) y los sitios críticos (suck sites) ${ }^{36}$.

\footnotetext{
32 WIPO Overview of Domain Name Decisions, http://www.wipo.int/amc/en/domains/search/overview2.0/ (última visita 30 de octubre 2018).

33 Por ejemplo, véase los casos The Royal Bank of Scotland Group plc, National Westminster Bank Plc A/K/A NatWest Bank v. Personal and Pedro Lopez, WipO Case No D2003-0166; The First Baptist Church of Glenarden v. Melvin Jones, WiPO Case No D2009-0022; Futbol Club Barcelona C. B.P, WIPO Case No D2014-1189.

34 Véase los casos Howard Jarvis Taxpayers Association v. Paul McCauley, WiPO Case No D20040014; Sutherland Institute v. Continuative LlC, WIPO Case No D2009-0693; Osunasport S.L. C. Wholsguard Protected / Pedro L. González Silva, WiPO Case No D2014-0367; Helena Revoredo Delvecchio, Prosegur Compañía de Seguridad, S.A. c. Raquel Nieto Mena, WiPO Case No D2018-0026.

35 Simon (2012).

36 Popović, Besarović (2005) p. 44.
} 
Vale decir que el término "suck" no es decisivo ${ }^{37}$ y que puede variar dependiendo del idioma utilizado (por ejemplo, jeboycotte en francés o scheisse en alemán) ${ }^{38}$.

Puesto que los sitios críticos están creciendo en su número, especialmente los de habla inglesa, en la literatura ya se estableció un estándar de denominar estos sitios como suck sitios ${ }^{39}$.

A pesar de que la política de la ICANN no debe ser utilizada para apagar el debate y la crítica, la mayoría de los panelistas encuentra que los nombres de dominio que consisten en una combinación de la marca comercial con algunas palabras negativas o peyorativas son confusamente similares. Por tanto, para los panelistas, un nombre de dominio que contiene el nombre de marca comercial es la evidencia prima facie de que el nombre de dominio fue registrado de mala fe.

Los argumentos más frecuentes para defender tal postura son las siguientes: a) que las personas que no hablan inglés no entenderán el sentido peyorativo de la palabra, solo se darán cuenta del signo distintivo en el nombre de dominio ${ }^{40}$; b) que los usuarios no van a entender que se trata de una página web con la cual el titular de la marca no está vincula$\mathrm{do}^{41}$; c) cuando un usuario escribe solo el nombre de la marca, el navegador web también insertará en los resultados este tipo de páginas, lo que dará lugar a confusión ${ }^{42}$; que la adición de un nombre de dominio a una marca comercial hace que sea automáticamente confusamente similar, independientemente de otros términos presentes en el nombre de dominio ${ }^{43}$; que en ciertas ocasiones particulares la palabra "suck" puede no tener un sentido peyorativo sino un propósito bastante descriptivo ${ }^{44}$; que la palabra "suck" es simplemente una adición genérica a la marca distintiva ${ }^{45}$.

En algunos casos, la facilidad con la que los panelistas transfieren el nombre de dominio al demandante, sin una apropiada averiguación para demostrar que las actividades de las partes demandadas coinciden con los requisitos previstos por la Política Uniforme es alarmante.

Con respecto a ese problema, en determinados casos se puede observar que la prueba de confusión bajo la política de la ICANN requiere solo el menor grado de confusión, comparable con la doctrina desarrollada por las Cortes estadounidenses, conocida como

\footnotetext{
37 En habla inglesa existen otros ejemplos como "fail", "gripe", "fuck", "wtf".

38 Marzetti (2005) p. 16.

39 En este sentido véase Dinwoodie (2000) pp. 519-520; Froomkin (2002) pp. 614 y ss.; Lipton (2005) pp. 21 y ss.; Lipton (2010) pp. 459 y ss.; Norton (2012) pp. 149 y ss.; Sharrock (2001) pp. 834 y ss. Sorkin (2002) pp. 48-50; WOOdARD (2009) pp. 1198-1199.

40 ADT Services AG v. ADT Sucks.com, WIPO Case No D2001-0213.

${ }^{41}$ Direct Line Group Ltd, Direct Line Insurance plc, Direct Line Financial Services Ltd, Direct Line Life Insurance Company Ltd, Direct Line Unit Trusts Ltd, Direct Line Group Services Ltd v. Purge I.T., Purge I.T. Ltd, WIPO Case, No D 2000-0583.

42 Wal-Mart Stores, Inc. v. Walsucks and Walmarket Puerto Rico, WIPO Case No D20000477.

43 Wal-Mart Stores, Inc. v. Richard MacLeod d/bla For Sale, WIPO Case No D2000-0662.

44 Vivendi Universal v. Jay David Sallen and GO247.com, Inc., WIPO Case No D2001-1121.

45 Red Bull GmbH v. Carl Gamel, WIPO Case No D2008-0253; Streamtel Corporation SRL v. Ton Kamminga, WIPO Case No D2010-0423; HM Publishers Holdings Ltd v. Marcus Costa Camargo Peres, WIPO Case No D2013-1597; Philip Morris USA Inc. v. Computer Services, Inc. WIPO Case No D2017-0847.
} 
initial interest confusion, la cual se aplica incluso en los casos cuando hay una tenue confusión temporal, lo que está ampliando los límites del derecho marcario tradicional ${ }^{46}$.

Según la legislación estadounidense, interés inicial de confusión se define como "la intención de la persona de desviar a los consumidores de la ubicación en línea del propietario de la marca a un sitio accesible bajo el nombre de dominio que podría dañar la buena voluntad representada por la marca, ya sea para obtener un beneficio comercial o con la intención de empañar o desacreditar la marca, creando una probabilidad de confusión en cuanto a la fuente, patrocinio, afiliación o aprobación del sitio" ${ }^{47}$. Esta doctrina resulta aplicable incluso "a pesar de que ninguna venta actual sea efectuada en razón de la confusión" ${ }^{48}$. Reconociendo la confusión de interés inicial, los paneles administrativos han cambiado el énfasis de las demandas de marcas fuera de la cuestión de si un registro y uso confunden a si atraen el interés inicial. Como señala Goldman, dado que dicha doctrina carece de una definición rigurosa, una clara justificación y un estándar uniforme para analizar reclamaciones, su flexibilidad doctrinal conduce a consecuencias perniciosas ${ }^{49}$.

Por otra parte, no hay que desestimar que existen opiniones opuestas donde se considera que un nombre de dominio no es automáticamente idéntico, tampoco es confusamente similar a una marca por solo hecho de acompañarla por un término peyorativo.

En un caso, el panelista destacó lo siguiente: "La Política Uniforme tiene un alcance limitado. Tiene el propósito de proteger contra la infracción de la marca registrada, y no de proporcionar un remedio general para toda mala conducta relacionada con los nombres de dominio. Publicar el material difamatorio en un sitio Web no justificaría la revocación de un nombre de dominio bajo la Política Uniforme" ${ }^{\text {" }}$.

También, en la práctica, desde un inicio se planteó la pregunta de si en la determinación de la similitud del nombre de dominio y la marca se debería tener en cuenta el contenido de la página web. La opinión predominante es que no es necesario y que basta hacer una comparación. Sin embargo, es importante señalar que los nombres de dominio también pueden reflejar intereses distintos que no se encuentran en relación con intereses comerciales, por lo que no todos los nombres de dominio pueden realizar funciones de marca comercial. A pesar de que la función de la Política Uniforme es respetar el derecho

\footnotetext{
46 Estée Lauder Inc. v. estelauder.com, estelauder.net and Jeff Hanna, WiPO Case No D2000-0869; aARC Inc. v. Jayashankar Balaraman, WiPO Case No D2007-0578; Broan-Nutone, LlC v. Ready Set Sales, WIPO Case No D2010-0920; Philip Morris USA Inc. v. Ky Le, WIPO Case No D2017-0598.

47 Lanham ACT, 15 USC, ch $12 \$ 1125$ (d)(1)(B)(i)(V): "the person's intent to divert consumers from the mark owner's online location to a site accessible under the domain name that could harm the goodwill represented by the mark, either for commercial gain or with the intent to tarnish or disparage the mark, by creating a likelihood of confusion as to the source, sponsorship, affiliation, or endorsement of the site".

48 Brookfield Communications, Inc. v. West Coast Entertainment Corporation, 174 F.3d 1036 (9th Cir. 1999).

49 Goldman (2005) p. 507.

50 "The UDRP has a narrow scope. It is meant to protect against trademark infringement, not to provide a general remedy for all misconduct involving domain names. "Wal-MART STORES, INC. V. WALLMARTCANADASUCKS.COM AND KENNETH J. HARVEY, WIPO D2000-1104.
} 
marcario existente ${ }^{51}$, ocurre que los paneles revisan únicamente la similitud entre los elementos textuales de la marca y el nombre de dominio, descartando los factores tradicionales de protección de marcas, como el concepto de buena voluntad.

En muchos casos donde se perjudican los derechos de la libre expresión, los panelistas interpretan los términos que establecen el requisito de la similitud confusa con una marca de una manera mucho más amplia que el sistema de la justicia ordinaria. Tales posturas se tienden a justificar afirmando que el derecho de la libre expresión no se aplica en estos casos simplemente porque los titulares de los nombres de dominio podrían haber ejercido su libertad de expresión seleccionando un nombre de dominio diferente ${ }^{52}$.

Un ejemplo ilustrativo es el caso Chubb Security Australia Pty Ltd v Mr. Shahim Tahmasebi, donde el panelista falló contra el demandado subrayando que la intención del demandado a criticar es legítima, pero “...no es legítimo utilizar la propia marca del demandante como plataforma para criticar al propio demandante" 53 .

Consiguientemente, se hace evidente que la complejidad de la posición de los registrantes respecto a los derechos de la libre expresión queda bien reflejada en el hecho de que se están llevando a cabo al mismo tiempo procesos opuestos. Conforme a lo dicho, discutir el requisito de la confusión en los casos que incluyen las cuestiones relativas a la libertad de expresión inmanente supone la dificultad para discernir. Esto es evidente incluso en las situaciones donde un mismo proveedor de la resolución de la disputa está decidiendo sobre la petición del mismo demandado, sin lograr el consenso respecto al tema, lo que implica resultados opuestos ${ }^{54}$.

\footnotetext{
51 Final Report of the WiPO Internet Domain Name Process, $\$$ 34. "It is further recognized that the goal of this WIPO Process is not to create new rights of intellectual property, nor to accord greater protection to intellectual property in cyberspace than that which exists elsewhere. Rather, the goal is to give proper and adequate expression to the existing, multilaterally agreed standards of intellectual property protection in the context of the new, multijurisdictional and vitally important medium of the Internet and the DNS that is responsible for directing traffic on the Internet. The WIPO Process seeks to find procedures that will avoid the unwitting diminution or frustration of agreed policies and rules for intellectual property protection".

52 Véase por ejemplo el caso The Royal Bank of Scotland Group and National Westminster Bank V. Pedro Lopez and A\& A System Solutions and Alberto Rodriguez, WIPO Case No D 2002-0823.

53 “... it is not in this Panel's view legitimate to use the Complainant's own trademark as a platform for criticizing the Complainant itself”. ChubB SeCURtTy Australia PTY LtD V MR. Shahim TahmasebI, WIPO Case No D2007-0769.

54 Por ejemplo, comparar los casos WAL-MART StORES, INC. V. WALLMARTCANADASUCKS.COM (WIPO Case No 2000-1104) donde el Panel administrativo razonó que un nombre de dominio acompañado con la palabra "suck" jamás podría ser confusamente similar en virtud de la Política Uniforme, con el caso WAL-MART STORES, INC. V. WALSUCKS (WIPO Case No D2000-047) donde el panel administrativo designado por el mismo proveedor, el mismo año, consideró este tipo de registro como un ejemplo de registro en mala fe que puede ser decidido aplicando la Política Uniforme.
} 


\section{LA POLÍTICA UNIFORME ESTÁ DESCARTANDO DOCTRINA DE USO LEAL}

En virtud de la política de la ICANN, la libertad de expresión no representa el uso legítimo per $s^{55}$. No obstante, los regímenes jurídicos y las normativas nacionales existentes a nivel comparado generalmente protegen los actos netamente expresivos, incluyendo los casos que involucran el uso de las marcas comerciales.

Incluso en los casos de las marcas notoriamente conocidas, las cuales gozan de protección suplementaria, "usos leales nominativos" son permitidos, siempre y cuando el término de la marca es la única manera de identificar lo que se discute ${ }^{56}$. Esta doctrina no es conocida únicamente en la teoría estadounidense. La manifestación de ello se puede evidenciar en la decisión del Tribunal de Grande Instance de París que declaró que "para ser objetable, el uso de una marca debe hacerse con fines comerciales o publicitarios. Sin embargo, el uso en un objeto de información no constituye una infracción" ${ }^{57}$.

Pese a que la Política Uniforme está compuesta tanto por la normativa sustantiva como la procedimental, una de las principales deficiencias de este mecanismo es que no se indican en forma precisa todos los aspectos relativos a su aplicación. Principalmente, la renuencia a definir qué es un dominio ha tenido un impacto significativo en el desarrollo del derecho marcario y su priorización dentro de este mecanismo.

La aplicación de aquellos términos que pueden establecer las defensas por las cuales un registrante puede demostrar que tiene un interés legítimo en el nombre de dominio, en algunos casos puede resultar inadecuada debido a que se ofrecen listas breves de los comportamientos que pueden entrar dentro de la categoría, pero no se hacen otros intentos de definirlo expresamente. Por lo tanto, dichas listas no proporcionan una orientación adecuada.

A pesar de que las normas son excesivamente amplias, la ICANN nunca respondió a las demandas de ofrecer aclaraciones adicionales, solicitadas tanto por los registrantes de los nombres de dominio como por los representantes del lobby marcario, continuamente insistiendo que la resolución de las disputas dependerá de las circunstancias de cada caso ${ }^{58}$.

Por ello, hasta la fecha, el único punto relevante relativo a la selección y la aplicación de los principios legales en este procedimiento se encuentra en el artículo 15 (a) del Reglamento, el cual establece que un panel administrativo "decidirá una demanda basándose en las declaraciones y en los documentos entregados y de conformidad con la política, con el presente Reglamento y con cualquier regulación y principio de ley que considere pertinente".

\footnotetext{
55 Recuérdese que el único punto relevante representa el artículo 4 (a) (iii).

56 Sobre tema, véase Froomkin (2002) p. 615.

57 "Pour être répréhensible, l'usage d'une marque doit être fait à des fins commerciales ou publicitaires. En revanche, l'usage dans un but d'information ne constitue pas une contrefaçon". TGI Paris (22 févr. 1995).

58 Véase SECOND STAFF REPORT ON IMPLEMENTATION DOCUMENTS FOR THE UNIFORM Dispute Resolution POLICY "Staff believes that the issue of whether a pattern is present will depend on the fact pattern presented in each case and that, until experience is accumulated with the policy, prescribing a more detailed definition is not warranted".
} 
Dicho artículo es demasiado amplio. En este orden de cosas, las normas animan una amplia discreción del panel al momento de evaluar las normas y principios jurídicos que se pueden aplicar en un determinado caso. Además, el artículo 10 de dicho Reglamento permite que los paneles formulen sus propias normas sobre "la admisibilidad, relevancia, materialidad y peso de las pruebas". Esto es de crucial relevancia, dado que las normas y los principios jurídicos que los panelistas consideren pertinentes se aplicarán al evaluar los tres requisitos sustanciales y sus condiciones meritorias previstos dentro del artículo 4 (a) de la Política Uniforme. En base a ello, la vaga terminología contribuye a interpretaciones plurivalentes cuyo resultado son enfoques contradictorios adoptados por los paneles. Asimismo, la ausencia de garantías contra el uso indebido de la discrecionalidad o contra las decisiones inconsistentes ${ }^{59}$, aumenta la inseguridad jurídica.

Muchas de las inconsistencias en la práctica ocurren porque los panelistas, en su mayoría abogados, están influenciados por las legislaciones nacionales. Esta circunstancia puede señalar un sesgo importante, dado que se estima que los demandantes pueden verse tentados a elegir el proveedor de resolución de disputas en función de la aplicación de las normas que este aplicará. Dicho concepto se conoce como forum shopping y significa que los demandantes están intentando obtener los resultados a su favor, donde el proveedor que más les convenga ${ }^{60}$.

En este contexto, en práctica han surgido preocupaciones sobre el sesgo en los casos relativos a la libertad de expresión en la que participan los panelistas y las partes estadounidenses. De hecho, algunos estudios han demostrado que los demandados estadounidenses tienen un mayor nivel de protección en los casos relativos al uso leal de los nombres de dominio comparado con los demandados de otros países ${ }^{61}$.

$\mathrm{Al}$ priorizar a los estadounidenses hay que tener en cuenta que "las leyes que protegen y las sensibilidades que rodean la libertad de expresión no son exclusivos de los Estados Unidos de América"62. Sin embargo, la defensa de uso leal tiende a ser ignorada por los panelistas de otros países.

Asimismo, como ya hemos mencionado, los panelistas provenientes de los países que desconocen la dilución pueden interpretar este instituto de forma mucho más amplia de lo que ha sido interpretado por los tribunales estadounidenses. En algunos casos los paneles

\footnotetext{
59 Cabe aclarar que la Política Uniforme carece de apelación interna, por lo que se excluye cualquier posibilidad de desafiar las decisiones internamente y de corregir eventuales decisiones erróneas. Del mismo modo, no existe ninguna sanción para los panelistas que están aplicando dicha política de forma incorrecta o cuando están ampliando el alcance de la propia política.

${ }^{60}$ Según el Reglamento de la Política Uniforme la elección de la institución donde se llevará a cabo el procedimiento administrativo la decide la parte que presenta la demanda (titular de la marca). Este hecho ha generado las preguntas sobre el sesgo a favor de los demandantes, lo que puede poner en cuestión la imparcialidad de los paneles. Sobre este tema consultar Froomkin (2002) pp. 641 y ss.; Geist (2002), Kesan, Gallo (2003) pp. 8 y ss.; SiMON (2012) pp. 69 y ss.

61 Sobre el tema véase SIMON (2012).

62 "Laws protecting and sensitivities surrounding freedom of speech are not unique to the United States". MATTHEW Harris, 1066 Housing Association Ltd. V. MR. D. Morgan, WIPO Case No D2007-1461.
} 
omiten determinar si la marca comercial en disputa es una marca famosa, no satisfaciendo un requisito esencial cuando se trata de la dilución ${ }^{63}$.

Tales decisiones expanden el alcance teórico de las disputas que deberían ser resueltas exclusivamente por la justicia ordinaria. Aunque conscientes de esto, determinados panelistas se justifican por la necesidad de aplicar la Política Uniforme uniformemente en todas las jurisdicciones.

Refiriéndose a las marcas famosas, a diferencia de las demandas de infracción de marcas, las acciones de dilución no dependen de un riesgo de confusión, sino se dirigen a una conducta que debilita el carácter distintivo o de prestigio asociado con una marca famosa ${ }^{64}$. Es importante señalar que la infracción de la marca en base a la dilución no está dentro de lo previsto por la Política Uniforme, es decir, se limita a los actos realizados con la intención de obtener una ganancia en el mercado, tal como se estableció en el Informe del Segundo Proceso de la OMPI relativo a los nombres de dominio de Internet. Sin embargo, la ICANN no ha empleado alguna medida concreta para promover esta postura.

Estas consideraciones son de especial relevancia teniendo en cuenta que la Política Uniforme carece de un mecanismo efectivo para obstaculizar el fenómeno de la ciberocupación inversa (reverse domain name hijacking), el cual ocurre cuando el propietario de una marca afirma los derechos de la marca sobre el nombre de dominio de otro usuario legítimo. En el caso de la ciberocuación inversa las únicas medidas previstas contra el titular de la marca suponen que el panel debería negarse a transferir el nombre de dominio al demandante, y que la demanda de mala fe debería publicarse ${ }^{65}$. En otras palabras, la ICANN atenuó el valor disuasivo dentro del Reglamento, ya que los titulares de las marcas no se pueden sancionar ni siquiera en las situaciones donde existe la evidencia clara de su mala fides.

De hecho, en el caso, Glimcher Univ. Mall v. GNO, Inc., el panel administrativo sostuvo que los remedios disponibles bajo la Política Uniforme sirven solo para el beneficio de los titulares marcarios ${ }^{66}$.

La decisión de la ICANN de ignorar este fenómeno ha creado una significativa fuente de inseguridad, visto que respecto a este tema los panelistas actúan motu proprio, de forma tal que la posición del demandado dentro del procedimiento llevado a cabo en virtud de la Política Uniforme es desventajosa. En algunos casos estas determinaciones están

\footnotetext{
63 InGersoll-Rand Co. v. Gully, WIPO Case No D2000-0021.

${ }^{64}$ En los Estados Unidos de América los titulares de marcas son capaces de demandar bajo la FeDERAL TRADEmark Dilution ACt (FTDA)-15 U.S.C. \$1125 (c) (2001) que contempla lo siguiente: “... el titular de una marca famosa que es distintiva... tendrá derecho a una orden judicial en contra de otra persona que, en comienza el uso de una marca o nombre comercial en el comercio que es probable que cause dilución por difuminación o dilución por degradación de la marca famosa, independientemente de la presencia o ausencia de confusión real o probable, de la competencia, o del daño económico real”.

"Subject to the principles of equity, the owner of a famous mark that is distinctive, inherently or through acquired distinctiveness, shall be entitled to an injunction against another person who, at any time after the owner's mark has become famous, commences use of a mark or trade name in commerce that is likely to cause dilution by blurring or dilution by tarnishment of the famous mark, regardless of the presence or absence of actual or likely confusion, of competition, or of actual economic injury".

65 Véase los artículos 15 (e) y 16 (b) del Reglamento para una Política Uniforme.

66 GLimcher UNIV. MALL V. GNO, INC., No FA0107000098010.
} 
alcanzando proporciones constitucionales, dado que se está decidiendo sobre cuestiones de competencia exclusiva de los tribunales.

\section{EL PROGRAMA DE NUEVOS DOMINIOS GENÉRICOS DE PRIMER NIVEL (gTLD)}

Como destacan Zalnieriute y Schneider políticamente hablando una de las funciones más importantes de la ICANN es decidir sobre la introducción de nuevos gTLD en el sistema de los nombres de dominio ${ }^{67}$.

Durante años la ICANN ha sido acusada de ocasionar artificialmente una escasez de los nombres de dominio disponibles y aumentar de esta manera la demanda en las ya repletas opciones de registración. Finalmente, el 20 de junio de 2011, la ICANN aprobó una resolución autorizando el Director Ejecutivo para introducir una multitud de nuevos nombres de dominios genéricos de primer nivel (gTLD) en el sistema DNS ${ }^{68}$. Dicha autorización fue sin precedentes, dado que, hasta enero de 2012, había solo veintidós gTLD aprobados.

El lanzamiento de nuevos dominios inmanente supone la expansión del sistema DNS. No obstante, conforme se van incorporando nuevos nombres de dominio, aumentan las posibilidades de los conflictos entre los nombres de dominio y las marcas comerciales.

Con el objetivo de salvaguardar los derechos de la propiedad industrial, la ICANN ha desarrollado mecanismos que permiten proteger los derechos marcarios durante la expansión del sistema a través de la Trademark Clearinghouse (TMCH).

La TMCH representa una base de datos de carácter global cuyo propósito es que los propietarios de marcas registradas en cualquier parte del mundo incluyan los datos de sus marcas registradas en una base centralizada, incluso antes del lanzamiento de los nuevos gTLD, así como durante los mismos, para poder disponer de un mecanismo de protección frente a cada tercera persona que registre un nombre de dominio en los nuevos gTLD que coincida con su marca registrada.

Este mecanismo tiene doble propósito. Uno es permitir a los titulares de las marcas inscritas en la TMCH optar por el registro de un determinado dominio durante el periodo sunrise $^{69}$, que les da acceso prioritario a los nombres de dominio en un nuevo gTLD. El segundo propósito se encuentra en la facultad del titular de la marca de recibir un aviso en el caso cuando un tercero solicite un gTLD correspondiente a su marca comercial, lo que permite al titular de la marca decidir si quiere proceder con el bloqueo de este dominio.

De lo anterior se colige que el nuevo sistema relativo a los gTLD incluye una serie de procedimientos mediante los cuales los propietarios de marcas no solo pueden proteger sus marcas contra la incursión en el nivel superior sin necesariamente tener que solicitar un

\footnotetext{
67 Zalnieriute y Schneider (2014) p. 15.

68 La información disponible en: https://www.icann.org/resources/board-material/resolutions-2011-06-20-en.

69 Un período Sunrise o de registro preferente se refiere al lanzamiento de un nuevo dominio de primer nivel (TLD) antes de que comience su registro público, lo que permite que los titulares de las marcas comerciales registren los nombres de dominios de su interés.
} 
gTLD, sino que también les permite indicar cuando creen que hay algo incorrecto respecto a la aplicación de un TLD $^{70}$.

Esto significa que el espacio de los nombres de dominio de internet tiende no solo a expandirse, sino también a contraerse. A propósito de esto, resulta imprescindible recordar que cada vez que la ICANN introduce nuevos nombres de dominio genéricos disponibles, a la vez restringe las condiciones para solicitarlos. Acotando las posibilidades de los usuarios a utilizar los nuevos espacios de los nombres de dominio, inevitablemente se genera escasez de los dominios disponibles.

Si bien los propietarios de marcas comerciales retienen los derechos legítimos para proteger sus valiosos recursos dentro de los nuevos gTLD, las nuevas posibilidades previstas por la ICANN, como el registro o la solicitud del bloqueo, suponen la probabilidad de perjudicar la libre expresión. Las políticas de la ICANN sobre la aprobación de solicitudes para nuevos gTLDs suponen un impacto significativo sobre quién puede usar esos nuevos dominios, en qué forma y, por ende, la accesibilidad de la información en internet ${ }^{71}$.

Dichos mecanismos amplían el alcance de la protección de la marca comercial más allá de lo previsto por las legislaciones nacionales. Inclusive, se genera un debate acerca de que estos mecanismos han alentado a las organizaciones intergubernamentales a pedir protección especial para sus acrónimos, lo que carece de fundamento jurídico ${ }^{72}$. Particularmente preocupante es que dentro del nuevo proceso relativo a los nuevos gTLD se han impulsado paralelamente los nuevos programas de registro que van más allá de lo previsto por los mecanismos de salvaguardias incorporadas en el mismo programa de nuevos gTLD de la ICANN ${ }^{73}$.

Como es bien conocido, bajo las legislaciones nacionales, el derecho marcario se basa en el principio de la especialidad de las marcas, lo que significa que la marca está protegida únicamente para los productos o servicios para los que fue registrada o para una determinada clase de productos o servicios con el cumplimiento de ciertas formalidades de carácter $\operatorname{administrativo~}^{74}$.

Además, los derechos de marca son basados en el principio de la territorialidad. El efecto más significativo de dicho principio es que se permite que diferentes personas sean

\footnotetext{
70 Lipton y Wong (2012) p. 27.

71 Contracting and the Registry Agreement, disponible en: https://newgtlds.icann.org/en/applicants/agb/ base-agreement-contracting\#stats.

$72 \mathrm{La}$ información disponible en: https://www.icann.org/en/system/files/correspondence/crocker-to-austin-etal-04oct16-en.pdf.

73 Uno de ellos, Donut Inc., ha proporcionado a los titulares de las marcas oportunidad única de bloquear los errores ortográficos de sus marcas a través del DPML (Domains Protected Marks List) Plus. Consultar aquí: http://www.donuts.domains/services/dpml-plus/dpml-plus-overview.

${ }^{74}$ Es importante tener en cuenta que en el derecho comparado existen dos formas de adquisición de la marca dependiendo si se trata de los procedimientos formales o informales dentro de diferentes sistemas jurídicos. En el sistema basado en el registro, la creación y la extinción de los derechos marcarios dependen de la inscripción en un registro nacional o regional pertinente, mantenido por la autoridad competente. En el sistema common law se diferencian la registered trademark (la marca comercial registrada) y la common law trademark, el cual puede incluir los nombres comerciales y personales. Cuando se trata de una marca common law, el demandante debe demostrar que ha cumplido con las condiciones para la adquisición de la marca a través del uso.
} 
titulares de marcas idénticas o similares para los productos o servicios idénticos o similares en diferentes jurisdicciones.

Del mismo modo, la defensa del uso equitativo permite que terceras personas utilicen una marca para los propósitos de la crítica, la parodia, la referencia en la literatura, así como en otros usos pertinentes siempre y cuando no se intente confundir a los consumidores. Estas limitaciones del derecho marcario son ignoradas por los mecanismos establecidos por la ICANN.

Asimismo, cabe decir que el sistema de la TMCH relativo a los nuevos gTLD supone que los solicitantes seleccionados pueden optar por llevar a cabo su registro de manera abierta o cerrada. Los registros cerrados están disponibles solo para un tipo específico de usuarios de internet. En este entorno la ICANN ha emprendido iniciativas que pueden perjudicar el derecho a la libertad de expresión.

Por ejemplo, en febrero de 2015, el Comité del Programa de nuevos gTLD de la ICANN determinó que la extensión .doctor supone un espacio restringido para los profesionales de la salud. Esta decisión de excluir una serie de usos posibles del nombre de dominio .doctor, incluyendo una amplia gama de individuos que poseen un doctorado profesional o incluso los titulares de las marcas como "Lawn Doctor", "Screen Doctor" o "Rug Doctor" se ha considerado como una medida contra los legítimos usuarios de la libertad de expresión. Incluso, parecía extraño que la ICANN de repente decidiera limitar a dominio .doctor, mientras al mismo tiempo los términos análogos, como .clinic se registraban en forma abierta.

Respecto a esto, hay que tener en cuenta que la comunidad de la GNSO $^{75}$ trató de proteger los derechos de la libre expresión en el programa de nuevos dominios gTLD al incluir los principios y recomendaciones respecto a la libertad de expresión en su Política Final aprobada en $2007^{76}$. En este marco, cabe mencionar los siguientes principios y recomendaciones que demuestran la intención explícita de proteger la libre expresión en el nuevo programa de la ICANN:

Principio G: "El proceso de evaluación de parámetros no debe infringir los derechos de libertad de expresión del solicitante que están protegidos por principios de derecho internacionalmente reconocidos" 77 .

En el mismo sentido, la Recomendación 3 contempla lo siguiente: "Los parámetros no deben infringir los derechos legales de otros que son reconocidos o ejecutables bajo principios de derechos generalmente aceptados e internacionalmente reconocidos. Ejemplos de estos derechos legales internacionalmente reconocidos incluyen, entre otros, los derechos definidos en el Convenio de París para la Protección de la Propiedad Industrial (en

\footnotetext{
75 La GNSO (Generic Names Supporting Organization) u la Organización de Apoyo para Nombres Genéricos es un subgrupo de la ICANN que se desempeña como una organización de apoyo para el desarrollo de políticas, asesoramiento a la Junta Directiva de la ICANN sobre las políticas relacionadas con el DNS, específicamente sobre la incorporación de nuevos dominios de nivel superior. Este cuerpo previamente era conocido como la Domain Name Supporting Organization (DNSO) que fue sustituida en 2003.

76 ICANN Generic Supporting Organisation, FINAL REPORT OF THE INTRODUCTION OF NEW GENERIC TOP-LEVEL DomaINS (2007).

77 "The string evaluation process must not infringe the applicant's freedom of expression rights that are protected under internationally recognized principles of law".
} 
particular los derechos de marcas), la Declaración Universal de los Derechos Humanos y el Pacto Internacional de Derechos Civiles y Políticos (en particular los derechos de libertad de expresión $)^{78}$.

Sin embargo, aunque la Política Final de la GNSO fue el resultado de años de arduo trabajo y compromisos hechos por todos los integrantes, esta posteriormente ha sido infringida por el mismo personal de la ICANN.

No solo que las recomendaciones finales que protegen la libertad de expresión fueron rápidamente ignoradas, sino en su lugar la Junta Directiva de la ICANN agregó un nuevo requisito a la política, denominado como Public Interest Commitments o "PICs" el cual representa los términos contractuales que la ICANN impone a los registros de los nuevos gTLD que contradicen las cláusulas destinadas a preservar los derechos de libre expresión de los usuarios de internet. Al adoptar los PIC, la ICANN establece un precedente peligroso despreciando por completo las protecciones de libre expresión que la comunidad de la GNSO pretendió incorporar en la nueva política de los gTLD.

\section{LAS POLÍTICAS RELATIVAS A LA ADMINISTRACIÓN DEL SISTEMA DNS IGNORAN LAS CUESTIONES RELATIVAS A LA LIBERTAD DE EXPRESIÓN}

Poco interés en temas relativos a la libre expresión es la consecuencia histórica en el proceso de protección de los intereses marcarios en internet. Dado que el primer borrador de la Política Uniforme fue elaborado por la OMPI (Organización Mundial de la Propiedad Intelectual), la posición desigual entre las partes dentro de este mecanismo se estableció desde un comienzo. Principalmente, esto se debe al hecho de que ante las amenazas de los ciberpiratas la preocupación fundamental de la OMPI fue la protección de las marcas, y no la protección contra el eventual abuso por parte de los titulares de las marcas.

En consecuencia, el Informe Final de la OMPI presentado el 30 de abril de 1999 sugiere que la Política Uniforme no solo se limita a los casos de registros y usos en mala fe, sino que además se aplica exclusivamente a los casos relacionados con la infracción de los derechos marcarios, excluyendo el resto de los signos distintivos ${ }^{79}$.

Siguiendo esta línea, las cuestiones relativas a los derechos y el uso de nombres de dominio se establecieron en el Informe del Segundo Proceso de la OMPI relativo a los nombres de dominio de internet, el cual describe los tipos de disputas a las cuales se puede aplicar este mecanismo de solución de controversias, descartando expresamente la interacción en la libre expresión. Se destacó que "la Política relega todas las disputas 'legítimas' -tales como aquellas en los que ambas partes tienen los derechos de marca de larga data en el nombre cuando se registró como un nombre de dominio- a los tribunales; solo los casos

\footnotetext{
78 "Strings must not infringe the existing legal rights of others that are recognized or enforceable under generally accepted and internationally recognized principles of law. Examples of these legal rights that are internationally recognized include, but are not limited to, rights defined in the Paris Convention for the Protection of Industry Property (in particular trademark rights), the Universal Declaration of Human Rights (UDHR) and the International Covenant on Civil and Political Rights (ICCPR) (in particular freedom of expression rights)".

79 Final REPORT of the WIPO INTERNET DOMAIN NAME PROCESS (1999).
} 
de registros abusivos están destinados a ser objeto de procedimiento administrativo de solución de controversias" $"$.

Como podemos observar, desde un inicio, los diseñadores de la Política Uniforme tenían previsto establecer un mecanismo de solución de controversias de alcance limitado, exclusivamente para combatir los casos de la ciberocupación.

A pesar de esto, en la abrumadora mayoría de los casos que implican el derecho a la libre expresión no se refleja esta idea.

No obstante, estos resultados indican un problema más grave, ya que están contrarios a los principios generales del derecho, así como a la constitucionalidad de los ordenamientos jurídicos nacionales. Generalmente, las Constituciones ex jure reconocen y protegen los derechos a expresar y difundir libremente los pensamientos, ideas y opiniones.

Cabe señalar que la reciente transición de custodia de las funciones de la IANA a la ICANN no parece aliviar este problema ${ }^{81}$. A pesar de ser formalmente considerado, el derecho de la libre expresión oculta profundos temas internos dentro del dicho proceso.

El proceso de transición incluye la adopción de un estatuto que afirma el compromiso de la ICANN para respetar los derechos humanos, incluyendo el establecimiento de un marco adecuado para la aplicación de este compromiso en sus políticas y procedimientos ${ }^{82}$.

Por otra parte, dentro de la Sección 1.2 (b) (viii) relativa a los valores fundamentales que deben guiar las decisiones y acciones de la ICANN se prevé que esto debe ser "sujeto a las limitaciones establecidas en la Sección 27.2, dentro del alcance de su Misión y otros Valores Fundamentales, respetando los derechos humanos internacionalmente reconocidos como lo requiere la ley aplicable. Este valor fundamental no crea, y no se interpretará para crear, ninguna obligación para la ICANN fuera de su misión, o más allá de las obligaciones establecidas en la ley aplicable. Este valor básico no obliga a la ICANN a hacer cumplir sus obligaciones en materia de derechos humanos o las obligaciones de otras partes en materia de derechos humanos contra otras partes" ${ }^{33}$.

\footnotetext{
80 Véase SeCONd StafF Report on Implementation Documents for the UNIform Dispute Resolution Policy 4.1. c) The Recommended Policy Is Minimalist in its Resort to Mandatory Resolution. "The policy relegates all "legitimate" disputes-such as those where both disputants had longstanding trademark rights in the name when it was registered as a domain name-to the courts; only cases of abusive registrations are intended to be subject to the streamlined administrative dispute-resolution procedure". El texto disponible en: http://archive.icann.org/en/udrp/ udrp-second-staff-report-240ct99.htm.

81 La discusión completa de esta transición está fuera del alcance de este artículo. Sin embargo, es importante entender que dichas funciones son claves para poder administrar internet y habitualmente se dividen en tres áreas: a) asignaciones de protocolos (IP), b) recursos numéricos de internet y c) gestión de la zona raíz. Para mayores detalles puede consultar LAKETIĆ (2016).

82 BYLAWS For INTERNET CORPORATION For ASSIGNED NAMES (2016).

83 Section 1.2 (b) (viii). Subject to the limitations set forth in Section 27.2, within the scope of its Mission and other Core Values, respecting internationally recognized human rights as required by applicable law. This Core Value does not create, and shall not be interpreted to create, any obligation on ICANN outside its Mission, or beyond obligations found in applicable law. This Core Value does not obligate ICANN to enforce its human rights obligations, or the human rights obligations of other parties, against other parties. Bylaws For Internet Corporation For Assigned Names And Numbers, A California Nonprofit Public-Benefit Corporation (draft dated May 26, 2016), p. 5.
} 
De conformidad con la Sección 27.2 (a), el reglamento propuesto no entraría en vigor o producirá efectos: “... menos que y hasta que se establezca un marco de interpretación de los derechos humanos ("FOI-HR").

(I) aprobado para ser presentado a la Junta por el CCWG-Accountability [grupo de trabajo] como una recomendación de consenso en el Work Stream 2, con las CCWG Chartering Organizations teniendo el rol descrito en la CCWG-Accountability Charter y

(II) aprobado por la Junta Directiva, en cada caso, utilizando el mismo proceso y criterios que para las Work Stream 1 Recommendations" 84 .

Este reglamento ambiguo define que el compromiso de la ICANN con los derechos humanos se deja para algún momento en el futuro y que no entrará en vigor hasta que la Junta Directiva de la ICANN apruebe el contenido y el alcance de ese compromiso.

Por ahora parece claro que la ICANN no tendrá ninguna obligación de responder a las violaciones de derechos humanos, incluyendo de cualquiera de las partes a las cuales ha delegado alguna de sus tareas, como sus administradores, registros, registradores, registrantes, entre otros.

\section{CONCLUSIÓN}

A lo largo del presente trabajo hemos observado diversos problemas en el reconocimiento del estatus normativo de las normas internacionales relativas a la libre expresión, así como obstáculos para su implementación integral en las sentencias en virtud de la Política Uniforme, lo que nos demuestra que la creciente importancia internacional de internet y decreciente papel del Estado nos obliga a reconsiderar la responsabilidad que cabe al sector privado respecto a la violación de derechos humanos en la red. Al priorizar los problemas causados por el hecho de que el sistema de los nombres de dominio de internet no estaba previsto para enfrentarse a cuestiones relacionadas con la propiedad industrial, los mecanismos alternativos de solución de controversias, los cuales priman en esta materia, son el reflejo de la lucha exclusiva contra la ciberocupación.

Por tanto, la falta de atención a las cuestiones de nombres de dominio que abarcan los temas más allá de su correlación con la ciberocupación, quiere decir, con las marcas comerciales, se ha convertido en una regla general. Esta perspectiva representa una visión estrecha de las correlaciones jurídicas provenientes de la aplicación de internet.

Sostenemos que, al priorizar las necesidades del mercado, no se puede perder de vista que actualmente los nombres de dominio a menudo reflejan intereses que no se encuentran en relación con los intereses marcarios, tampoco comerciales, sino, con la libertad de expresión.

Como hemos explicado, los mecanismos de protección de los nombres de dominio de la ICANN exceden los derechos conferidos por la marca y tienen implicaciones preocupantes. Las decisiones exorbitantes se han transformado en medios para suprimir la libertad

84 "... unless and until a framework of interpretation for human rights ("FOI-HR") is (i) approved for submission to the Board by the CCWG-Accountability [working group] as a consensus recommendation in Work Stream 2, with the CCWG Chartering Organizations having the role described in the CCWG-Accountability Charter, and (ii) approved by the Board, in each case, using the same process and criteria as for Work Stream 1 Recommendations". p. 137. 
de expresión y desintegrar los derechos de los registrantes de los nombres de dominio. Una visión demasiado estrecha respecto al requisito de la confusión similar amenaza la defensa del uso leal de los registrantes de los nombres de dominio, lo que lleva al incumplimiento de la protección concebida por las legislaciones nacionales. A modo que los registrantes están sometidos a tratamientos diferentes, lo que se refleja en la heterogeneidad de las decisiones en que diferentes paneles consideran reclamaciones de la libertad de expresión, se ha dado la inconsistencia en un ámbito del derecho que se está volviendo cada vez más sensible en el entorno de un nuevo forum de crítica, como es el caso de internet.

Del mismo modo, la ICANN como una institución de gobernanza global de internet debería actuar en interés público. De hecho, en los artículos de Actas Constitutivas de la ICANN se establecía que: "La Corporación operará en beneficio de la comunidad de internet en su conjunto, llevando a cabo sus actividades de conformidad con los principios relevantes del derecho internacional, las convenciones internacionales aplicables y las leyes locales y, en la medida apropiada y consistente con estos Artículos y sus Estatutos, mediante procesos abiertos y transparentes que permitan la competencia y libre ingreso a los mercados relacionados con Internet. A este efecto, la Corporación cooperará, según corresponda, con las organizaciones internacionales pertinentes" ${ }^{\prime 5}$.

Sin embargo, la ICANN ha reformado Actas Constitutivas y ya no funciona al margen del marco del ordenamiento jurídico internacional ${ }^{86}$. Como hemos discutido, el Estatuto actual de la ICANN atribuye un ámbito distinto de competencias ${ }^{87}$.

Incluso antes de estas reformas, las crecientes demandas de proteger los derechos en internet, en particular la libertad de expresión, no han dado frutos. Cuando la NTIA aún administraba el contrato con la ICANN, lo que significa que estaba sujeta a las mismas restricciones y obligaciones legales para proteger la libertad de expresión que el gobierno estadounidense, algunas de las partes interesadas más influyentes se resistían a comprometer a la ICANN con cualquier obligación de proteger los derechos humanos. La Política Final de la GNSO relativa a la protección de la libre expresión fue infringida por el mismo personal de la ICANN.

Jurídicamente hablando, no existe ningún respaldo para decidir en función de motivos comerciales al anteponer los derechos de la propiedad industrial a las exigencias de la libertad de expresión.

Estamos enfrentándonos al riesgo de atenuar lo que no es atenuable, la severidad de la norma básica de derechos humanos. Si aceptamos disminuir la responsabilidad del sector privado tolerando la aplicación de patrones voluntarios respecto a la libre expresión, estamos perdiendo de vista que "la propiedad intelectual es solo un pequeño lago en el con-

\footnotetext{
85 "The Corporation shall operate for the benefit of the Internet community as a whole, carrying out its activities in conformity with relevant principles of international law and applicable international conventions and local law and, to the extent appropriate and consistent with these Articles and its Bylaws, through open and transparent processes that enable competition and open entry in Internet-related markets. To this effect, the Corporation shall cooperate as appropriate with relevant international organizations". ARTículos DE ACTAS CONSTITUTIVAS DE LA ICANN (2012): artículo 4 .

86 La información está disponible en: https://pti.icann.org/articles-of-incorporation.

87 Tener en cuenta Bylaws For InTERnet Corporation For Assigned NAMES AND Numbers (2016).
} 
tinente más grande; es un conjunto de excepciones limitadas y tentativas a derechos mucho más grandes y fundamentales como el derecho a la libertad de expresión" ${ }^{\prime 8}$.

\section{BIBLIOGRAFÍA CITADA}

Bone, Robert (2006): "Hunting goodwill: a history of the concept of goodwill in trademark law", Boston University Law Review, vol. 86: pp. 547-622.

BREAKEY, Hugh (2016): Intellectual liberty: Natural rights and intellectual property (Abingdon and New York, Routledge, primera edición).

Bygrave, Lee (2015): Internet governance by contract (Oxford, Oxford University Press, primera edición).

Dinwoodie, Graeme (2000): “(National) Trademark Laws and the (Non-National) Domain Name System”, University of Pennsylvania Journal of International Economic Law, vol. 21, N 3: pp. 495-521.

Dinwoodie, Graeme y Janis Mark (2008): Trademark law and Theory: A handbook of contemporary research (Cheltenham, Edward Elgar Publishing, primera edición).

Froomkin, Michael (2002): “Icann's Uniform Dispute Resolution Policy-Causes and (Partial) Cures”, Brooklyn Law Review, vol. 67, N³: pp. 605-718.

FroOMKIN, Michael y LEMLEY Mark (2003): "ICANN and anti-trust", University of Illinois Law Review, vol. 1: pp. 1-76.

GeIsT, Michael (2002): "Fair. Com: An examination of the allegations of systemic unfairness in the ICANN UDRP”, Brooklyn Journal of International Law, vol. 27, $\mathrm{N}^{\circ} 3$ : pp. 903-938.

Goldman, Eric (2005): "Deregulating relevancy in Internet trademark law", Emory Law Journal, vol. 54, $\mathrm{N}^{\circ} 1$ : pp. 507-596.

Hoffman, Paul (2012): "The tao of IETF: A novice's guide to the Internet Engineering Task Force”. Disponible en: https://www.ietf.org/about/participate/tao/. Fecha de consulta: 9 de septiembre de 2018.

Hofmann, Jeanette (2016): "Multi-stakeholderism in Internet governance: putting a fiction into practice", Journal of Cyber Policy, vol. 1, No 1: pp. 29-49.

Hofmann, Jeanette; Katzenbach, Christian y Gollatz, Kirsten (2017): "Between Coordination and Regulation: Finding the Governance in Internet Governance", New Media \& Society, vol. 19, Nº 9: pp. 1406-1423.

Johnson, Eric (2012): "Intellectual Property and the Incentive Fallancy", Florida State University Law Review, vol. 39, $\mathrm{N}^{\circ}$ 3: pp. 623-679.

Kesan, Jay y Gallo, Andres (2003): "ICANN / UDRP Performance-An Empirical Analysis”, Working Papers 03-08, NET Institute. Disponible en: http://www.netinst. org/KesanGallo.pdf. Fecha de consulta: 7 de diciembre de 2018.

KLEINWÄCHTER, Wolfgang (2000): "Icann as the United Nations of the Global Information Society? The Long Road Towards Self-regulation of the Internet", International Communication Gazette, vol. 62, № 6: pp. 451-476.

${ }^{88}$ BREAKEY (2016) p. 2 
LAKETIĆ, Jelena (2016): "Control de Internet: Las implicaciones políticas de las funciones de la IANA", Revista Chilena de Derecho y Tecnologia, vol. 5, N²: pp. 127-151.

Lipton, Jacqueline (2005): "Beyond Cybersquatting: Taking Domain Name Disputes Past Trademark Policy”, Wake Forest Law Review, vol. 40, N 4: pp. 1361-1425.

Lipton, Jacqueline (2010): "Bad Faith in Cyberspace: Grounding Domain Name Theory in Trademark, Property, and Restitution”. Harvard Journal of Law and Technology, vol. 23, $\mathrm{N}^{\circ} 2$ : pp. 447-481.

Lipton, Jacqueline y Wong, Mary (2012): “Trademarks and freedom of expression in ICANN's new gTLD process", Monash University Law Review, vol. 38, $\mathrm{N}^{\circ} 1$ : pp. 188-228.

MarzetTi, Maximiliano (2005): “Speechless Trademarks? Dilution Theory Meets Freedom of Speech", WIPO Collection Paper.

MCCARTHY, Thomas (1996): McCarthy on trademarks and unfair competition (Deerfield, IL, Clark Boardman Callaghan, cuarta edición).

McKenna, Mark (2007) "The normative foundations of trademark law", Notre Dame Law Review, vol. 82, No 5: pp. 1839-1916.

Mueller, Milton (2002): Ruling the Root: Internet Governance and the Taming of Cyberspace (Cambridge, Massachusetts and London, MIT Press, primera edición).

Mueller, Milton (2010): Networks and States: The Global Politics of Internet Governance (Cambridge, Massachusetts and London, MIT Press, primera edición).

NorTon, Ben (2012): "Constitutional Internationalization of ICAAN's UDRAP”, Arizona Journal of International and Comparative Law, vol. 29, $\mathrm{N}^{\circ}$ 1: pp. 137-173.

Nunziato, Dawn (2003): "Freedom of Expression, Democratic Norms, and Internet Governance”, Emory Law Journal, vol. 52, № 1: pp. 187-306.

Popović, Dušan y Besarović, Vesna (2005): Imena internet domena i pravo intelektualne svojine (Belgrado, Institut za uporedno pravo, primera edición).

Powers, Shawn y Jablonski, Michael (2015): The Real Cyber War: The Political Economy of Internet Freedom (Urbana, Chicago and Springfield, University of Illinois Press, primera edición).

Sharrock, Lisa (2001): "The Future of Domain Name Dispute Resolution: Crafting Practical International Legal Solutions from Within the UDRP Framework", Duke Law Journal, vol. 51, $\mathrm{N}^{\circ}$ 2: pp. 817-849.

Simon, David (2012): "An Empirical Analysis of Fair Use Decisions under the Uniform Domain-Name Dispute-Resolution Policy”, Boston College Law Review, vol. 53, $\mathrm{N}^{\circ} 1$ : pp. 64-129.

Sorkin, David (2002): "Judicial review of ICANN domain name dispute decisions", Santa Clara Computer \& High Technology Law Journal, vol. 18, № 1: pp. 35-55.

WeINBERG, Jonathan (2000): "ICANN and the problem of legitimacy", Duke Law Journal, vol. 50, N 1: pp. 187-260.

WoOdard, Elizabeth (2009): "The UDRP, ADR, and Arbitration: Using Proven Solutions to Address Perceived Problems with the UDRP”, Fordham Intellectual Property, Media \& Entertainment Law Journal, vol. 19, № 4: pp. 1169-1213. 
Zalnieriute, Monika y Schneider, Thomas (2014): “ICANN's Procedures and Policies in the Light of Human Rights, Fundamental Freedoms and Democratic Values". Consejo de Europa. Disponible en: https://papers.ssrn.com/sol3/papers. cfm?abstract_id=2667478.

\section{TRATADOS, INSTRUMENTOS E INFORMES INTERNACIONALES}

Artículos de Actas Constitutivas de la ICANN disponible en: https://www.icann.org/ resources/pages/articles-2012-02-25-en. Fecha de consulta: 23 de mayo de 2017.

Bylaws For Internet Corporation For Assigned Names And Numbers, A California Nonprofit Public-Benefit Corporation (May 27, 2016). Disponible en: https://www. icann.org/en/system/files/files/adopted-bylaws-27may16-en.pdf. Fecha de consulta: 15 de diciembre de 2016.

Declaración Universal de los Derechos Humanos. Resolución 217 A (III) de la Organización de las Naciones Unidas, París, Francia, 10 de diciembre de 1948.

Final Report of the WiPO Internet Domain Name Process. (April 30, 1999).

iCANN Generic Supporting Organisation, Final Report of the Introduction of New Generic Top-Level Domains (August 8, 2007). Disponible en: https:/gnso.icann.org/ en/issues/new-gtlds/pdp-dec05-fr-parta-08aug07.htm. Fecha de consulta: 24 de marzo de 2017.

Política UNIFORME PARA LA RESOlUCión DE CONFLICTOS EN MATERIA DE NOMBRES DE DOMINIO, aprobada por la Corporación de Asignación de Nombres y Números de Internet (ICANN) el 24 de octubre de 1999.

Reglamento para una Política uniforme de Resolución de disputas sobre Nombres de DomINIO, aprobado por la Junta directiva de la ICANN el 30 de octubre de 1999.

Second Staff Report on Implementation Documents for the Uniform Dispute Resolution Policy (October 24, 1999).

U.N. Special Rapporteur on the Promotion and Protection of the Right to Freedom of Opinion and Expression, Report to the Human Rights Council, Seventeenth Session Agenda Item 3, United Nations General Assembly, U.N. Doc. A/HRC/17/27 (May 16, 2011) (by Frank La Rue). Disponible en: http://www2.ohchr.org/english/bodies/hrcouncil/docs/17session/A.HRC.17.27_en.pdf. Fecha de consulta: 12 de marzo de 2017.

U.S. Department of Commerce, Managment of Internet Names and Addresses, 63 Fed. Reg, 31, 741 (10 de junio de 1998). Disponible en: https://www.icann.org/resources/unthemed-pages/white-paper-2012-02-25-en. Fecha de consulta: 12 de diciembre de 2016.

\section{NORMAS LEGALES CITADAS}

Trademark Dilution Revision Act of 2006, Pub. L. No 109-312, 120 Stat. 1730 (codified as amended at 15 U.S.C.A. $\$ 1125(\mathrm{c})$ (West Supp. 2007)) (Estados Unidos de América). 


\section{JURISPRUDENCIA CITADA}

Brookfield Communications, Inc. V. West Coast Entertainment Corporation, 174 F.3d 1036 (9th Cir. 1999).

TGI PARIs, 22 FÉVRIER 1995: PIBD 1995, III, p. 257 (Francia).

Tribunal europeo de Derechos Humanos: Ahmet Yildirim v. Turkey, sentencia de 18 de diciembre de 2012, demanda No 3111/10.

\section{JURISPRUDENCIA ADMINISTRATIVA CITADA}

AARC INC. V. JAYASHANKAR BALARAMAN, WIPO Case No D2007-0578.

ADT Services AG v. ADT Sucks.com, WIPO Case No D2001-0213.

Chubb Security Australia Pty Ltd v Mr. Shahim Tahmasebi, WIPO Case No D2007-0769

Direct Line Unit Trusts Ltd, Direct Line Group Services Ltd V. Purge I.T., Purge I.T. LTD, WIPO Case, No D 2000-0583.

Fox NeWs Network, L.L.C. V. C\&D International Ltd. and Whois PrivaCy PRotection SERVICE, WIPO Case No D2004-0108.

GLIMCHER UNIV. MALL V. GNO, INC., No FA0107000098010.

InGersoll-Rand Co. v. Gully, WIPO Case D2000-0021 (9 de marzo de 2000).

HM Publishers Holdings Ltd. v. Marcus Costa Camargo eres, WIPO Case No D2013-1597.

Helena Revoredo Delvecchio, Prosegur Compañía de Seguridad, S.A. c. Raquel Nieto MENA, WIPO Case No D2018-0026.

VIVENDI UnIVERSAL V. JaY DaVId SALLEN and GO247.com, INC., WIPO Case No D2001-1121. Matthew Harris, 1066 Housing Association Ltd. V. Mr. D. Morgan, WiPO Case No D2007-1461.

Osunasport S.L c. WhoIsguard Protected / Pedro L. Gonzalez Silva, WipO Case No D2014-0367.

Philip Morris USA Inc. v. Computer Services, Inc., WiPO Case No D2017-0847.

Philip Morris USA INC. v. KY LE, WIPO Case No D2017-0598.

Sutherland Institute v. Continuative LLC, WIPO Case No D2009-0693.

Red Bull GmbH v. Carl Gamel, WIPO Case No D2008-0253.

Streamtel Corporation SRL v. Ton Kamminga, WIPO Case No D2010-0423.

The First Baptist Church of Glenarden v. Melvin Jones, WiPO Case No D2009-0022. Futbol Club Barcelona C. B.P, WIPO Case No D2014-1189.

The Royal Bank of Scotland Group and National Westminster Bank V. Pedro Lopez and A\&A System Solutions and Alberto Rodriguez, WiPO Case No D 2002-0823.

WaL-Mart Stores, INC. V. RICHARD MACLEOD D/B/A FOR SALE, WIPO Case No D2000-0662. WAL-MART StORES, INC. v. WALSUCKS, WIPO Case No D2000-047.

Wal-Mart Stores, Inc. V. Walsucks and Walmarket Puerto Rico, WipO Case No D20000477.

WAL-MART StORES, INC. V. WALLMARTCANADASUCKS.COM AND KENNETH J. HARVEY, WIPO D2000-1104. 